\title{
About the global effect and the critical role of retinal eccentricity: Implications for eye movements in reading.
}

\author{
Françoise Vitu \\ CNRS, Université de Provence
}

\begin{abstract}
In the present paper, I review evidence for the universality of the global effect, i.e. the general tendency to move the eyes towards the centre of gravity of the peripheral configuration, and show that the effect is strongly constrained by the retinal location of the stimuli. First, stimuli that are displayed in a central foveal region of a $1-1.5^{\circ}$ radius fail to deviate the eyes in a centre-of-gravity manner; this is referred to as the foveal dead zone. Second, the stimuli that are too eccentric relative to the saccade target and/or the main stimulation site are filtered out. These limitations reflect physiological constraints and the dynamics of the patterns of activity in a visual saliency map. They form the basis for a low-level centre-of-gravity type account of eye guidance in natural perceptual tasks such as reading.
\end{abstract}

\section{Keywords: Saccades, global effect, remote distractor effect, visual saliency, launch} site, reading

Over the last forty years, a large number of studies investigated saccadic performance (accuracy and latency) in multiple-stimulus visual displays. This research led to the discovery of the global effect and other related phenomena. As we shall see, the global effect is a very robust phenomenon which very likely reflects the properties of the saccadic system. After presenting recent data suggesting the critical role of stimulus eccentricity, I will propose that the mechanisms which underlie this effect may play a major role in reading.

\section{The global effect: A universal phenomenon.}

Coren and Hoenig (1972) were the first to show that saccadic eye movements are much less accurate when the saccade target stimulus (a red circle) is simultaneously presented with one or several distractors (black stimuli) than when the target is presented in isolation; in the former case, the eyes are deviated towards an intermediate location between the distractor(s) and the target. In 1982, Findlay demonstrated for the first time that this effect comes from a general tendency to direct the eyes towards the centre of gravity of the visual configuration formed by the peripheral stimuli. After replicating Coren and
Hoenig's findings, he conducted two critical experiments, where participants were presented simultaneously with two target objects in the periphery, that is, two empty squares of same or different sizes. Participants' task implicitly required precise fixations on individual targets since it consisted of determining whether there was a small gap in one of the squares. Still, the initial saccade invariably landed in between the two squares, and interestingly the deviation from the midpoint between the two was always towards the largest square of the two. On that basis, Findlay concluded that saccade amplitude is computed based on global visual integration processes in the periphery. He related these to the very large and overlapping receptive fields of the neurons at the level of the Superior Colliculus, a substrate involved in the generation of saccadic eye movements (Mcllwain, 1976, 1991).

Since then, the global effect has been replicated in many different laboratories, and in a wide variety of perceptual tasks, in both humans and monkeys. First, the effect was confirmed in different variants of the original paradigm, that is, a saccade target item was presented simultaneously with one or several distractors (Deubel, Findlay, Jacobs, \& Brogan, 1988; Eggert, Sailer, Ditterich, \& Straube, 2002; Ottes, Van Gisbergen, \& Egger- 
mont, 1984, 1985; Walker, Deubel, Schneider, \& Findlay, 1997; Weber, Latanov, \& Fischer, 1993), or two target elements were displayed simultaneously (Chou, Sommer, \& Schiller, 1999; Deubel, Wolf, \& Hauske, 1984; Edelman \& Keller, 1998; Findlay, Brogan, \& Wenban-Smith, 1993; see also Weber et al., 1993) and in some cases participants were explicitly asked to fixate each of the items accurately (Findlay \& Kapoula, 1992). In addition, the effect was found in visual search tasks (Arai, McPeek, \& Keller, 2004; Findlay, 1997; Findlay \& Gilchrist, 1997; McSorley \& Findlay, 2003; Zelinsky, Rao, Hayhoe, \& Ballard, 1997; see also Godjin \& Theeuwes, 2002), the free scanning of visual displays (Findlay, 2004; Findlay \& Brown, 2006; see also McGowan, Kowler, Sharma, \& Chubb, 1998; Melcher \& Kowler, 2001), the reading of isolated words (Vitu, 1991a), and in saccade-target tasks with reading-like stimuli (i.e. the target was a letter embedded in a meaningless string of consonants; Coëffé \& O'Regan, 1987; Jacobs, 1987; Vitu, Lancelin, Jean, \& Farioli, 2006). Note that most experiments were conducted on adults, but the data reported by Cohen and Ross (1978) suggest that the global effect is already at work in third grade children (about 8.5 years old). In their experiment, the target (a green LED) was presented simultaneously with two distractor stimuli (two red LEDs) at variable locations relative to the saccade target; on average, the eyes landed near the centre of the three-stimulus configuration.

In contrast with Findlay's (1982) original account of the global effect, several authors made the assumption that the effect reflects the use of visual strategies rather than being a default oculomotor response to the simultaneous presentation of peripheral stimuli. The eyes would be sent to the location in the array that is most appropriate for the task. In saccade target tasks, an intermediate position between the target and the distractor would be preferred because this would bring the eyes closer to the potential target location at least when this is not predictable (He \& Kowler, 1989; see also Jacobs, 1987), while in perceptual tasks, fixating the centre of the array would facilitate visual information intake (He \& Kowler, 1991; see also McConkie, Kerr, Reddix, \& Zola, 1988).

However, several findings argue against a strategybased account of the global effect. First, although the predictability of target location weakens the effect of distractor stimuli on saccade amplitude, it is not sufficient to cancel the effect, at least for normal-latency saccades
(Coëffé \& O'Regan, 1987; but see He \& Kowler, 1989); note also that in several experiments, the relative position of target and distractor stimuli was kept constant (e.g. the target was always to the right of the distractor; Vitu et al., 2006), and a global effect was still present. Second, when the relative luminance of distractor and target stimuli was manipulated, it was shown that the eyes were deviated towards the most luminous stimulus of the two (Deubel et al., 1984); the same was found when texture density was manipulated (Menz \& Gröner, 1987). These data do not only confirm that the effect is related to global visual integration processes (Findlay, 1982), but they also suggest, as noted by Deubel et al. (1988), that the eyes are not sent towards an optimal location for perceptual processing; otherwise, the eyes would move towards the least and not the most visible of the two stimuli. Actually, in several experiments, accurate saccades were required for the needs of the perceptual task, but the global effect was only slightly weakened (Findlay, 1982; Findlay \& Kapoula, 1992) or remained unaffected (Coren \& Hoenig, 1972). Furthermore, the effect did not vary as a function of the discriminability of the target in peripheral vision (Jacobs, 1987).

Of course, the global effect is not an irrepressible oculomotor response to multiple-stimulus displays. As the time for saccade programming increases, averaging becomes less likely (Chou et al., 1999; Coëffé \& O'Regan, 1987; Edelman \& Keller, 1998; Eggert et al., 2002; Findlay, 1982; Jacobs, 1987; McSorley \& Findlay, 2003; Ottes et al., 1985; Vitu et al., 2006; Weber et al., 1993; see also Findlay \& Blythe, in press; Godjin \& Theeuwes, 2002). However, saccades exclusively land on the target item only when saccade latencies are longer than about 200-300ms for visually dissimilar target and distractor stimuli that are three degrees apart (McSorley \& Findlay, 2003; see also Eggert et al., 2002). Furthermore, when the target is systematically the central letter of a letter string, and it is visually distinct from distractor letters (e.g. ' $x x x k x x x$ '), only saccades with a latency longer than about 300-600ms (depending on target eccentricity) accurately land on target (Vitu et al., 2006; see also Coëffé \& O'Regan, 1987; Jacobs, 1987).

Time-related changes in the likelihood of a global effect strongly argue for the assumption that averaging is a default option of the oculomotor system, and that it results from early and poorly resolved visual input. As noted above, the neurons at the level of the Superior Col- 
liculus are characterized with large and overlapping receptive fields and they very likely contribute to the effect; simultaneous activation of neighbouring sites would coalesce in a single, central peak, favouring in turn the execution of saccadic eye movements towards intermediate locations (Lee, Rohrer, \& Sparks, 1988; for a review see McIlwain, 1991). Deubel et al. (1988) noted that the Superior Colliculus is probably not the only brain centre involved and that global visual integration processes may not be as simple as initially envisioned by Findlay. They showed that when a distractor differs from the background only by the orientation of its constituent elements, it deviates the eyes in a manner very similar to as luminance-defined distractors (see also Findlay et al., 1993). Other findings however suggest that extraction of orientation and/or contour information may not be part of the spatial integration process (see Guez, Marchal, Le Gargasson, Grall, \& O'Regan, 1994; McGowan et al., 1998).

The level of visual processing involved in the global effect still remains very coarse in comparison with that associated with perceptual processes as suggested in several papers. Eggert et al. (2002) found a global effect in a saccade target task, but failed to find a similar effect for perceptual localization judgements. On the other hand, Jacobs (1987) reported that the likelihood of correctly localizing a target letter in a parafoveal letter string was greater for outer in comparison with inner letters, but when participants were asked to move their eyes to the target letter, the eyes landed to the left of the string's centre irrespective of target location; saccades were accurate only when saccade latency was greatly prolonged (about $600 \mathrm{~ms}$ ). Thus, for the eyes to be sent to a specific target location, the saccade programming time needs to be particularly long (Eggert et al., 2002), which suggests that selective eye guidance may only intervene at later visual processing stages or when visual information processing reaches sufficient detail (Ottes et al., 1985; Vitu et al., 2006; see also Coëffé \& O'Regan, 1987; Jacobs, 1987). I will come back to this point in the final section.

Note that interpretation of the global effect in terms of collicular-type processes may suggest that the effect is restricted to paradigms that involve stimulus onsets, as brain centres such as the Superior Colliculus are particularly sensitive to visual transients. However, I do not think this is a reasonable assumption. It is true that in most experiments the global effect characterized the initial saccade that was executed after display onset in either gap (mainly 0-ms gap durations) or overlap conditions. However, the effect was also observed in the absence of transient stimulation. For instance, in Findlay and Brown's (2006) study, averaging responses occurred after the initial saccade, while participants scanned an unchanging set of randomly-arranged ring stimuli and silently counted the number of target elements (i.e. a ring that contained a specific letter); the initial saccade in a trial always took the eyes to the upper left corner of the display where the identity of the target letter was indicated. These data show that the global effect is not an oculomotor reflex-like response to visual transients (see also Findlay \& Blythe, in press), and hence that it may well be at work in natural perceptual tasks such as reading.

I will further develop this key assumption below, but I will first review evidence for a differential role of distractor stimuli depending on their position on the retina.

\section{The critical role of retinal eccentricity}

In natural perceptual tasks such as reading, the retina receives simultaneously multiple visual inputs in foveal and peripheral regions, and we may wonder how the global effect operates in that framework. Indeed, if all stimuli were potential distractors, then the centre-ofgravity notion would make no sense at all. However, as suggested by several studies, stimuli contribute with differential weights depending on their size and their luminance (see above), as well as their position on the retina and with respect to the saccade target.

Walker et al. (1997) investigated in a simple saccade target task the role of distractor stimuli as a function of their eccentricity. In their experiment, participants were presented with either a single target stimulus or two simultaneous target and distractor stimuli. They found that distractor stimuli deviated the eyes from their saccade target only when they were displayed within a limited region around the saccade target, i.e. along the ispilateral target axis or within $20^{\circ}$ of the target axis (see also Findlay, 2004; Findlay \& Brown, 2006; Ottes et al., 1985). Distractors outside of this critical region did not affect saccade amplitude, but greatly delayed saccade onset; this was referred to as the remote distractor effect (see also Benson, this volume). 
As we shall see in the next two sections, the global effect may even operate in a smaller region (see also Findlay \& Blythe, in press), which excludes the stimuli displayed in a central foveal region (or dead zone), as well as the stimuli that are too eccentric with respect to the saccade target or the main site of activity.

\section{A foveal dead zone for the global effect}

In their original study, Walker et al. (1997) investigated the global and the remote distractor effects mainly with peripheral distractor and target stimuli, and when distractors were displayed in the foveal region, they appeared at fixation or contra-laterally to the saccade target. In one experiment only, the target (i.e. a cross) was displayed in the foveal region, while the distractor (i.e. a circle) appeared more peripherally along the ipsilateral target axis. Results suggested the presence of a global effect, but the effect was actually slightly smaller than when both target and distractor stimuli were presented in the periphery. In several recent studies, we reinvestigated this issue by testing the influence of foveal distractor stimuli on the accuracy of saccadic eye movements towards a more eccentric ipsilateral target. Overall, our findings suggest that stimuli displayed in the central foveal region fail to produce a global effect.

In our original study, we used complex reading-like distractor and target stimuli (Vitu et al., 2006). The target was the central letter of a letter string (e.g. 'xxxkxxx') displayed at a variable eccentricity to the right of fixation, and the distractor was an x-letter string of variable length (1 to 9 letters or .31 to 2.79 degrees). The first letter of the distractor string was always centred on the fixation point and other distractor letters extended to the right, thus in the same hemifield as the target letter string; note that target and distractor stimuli were always presented simultaneously with two other letter strings positioned to the right of the initial target letter string. As suggested by Walker et al.'s (1997) original findings, a global effect operates when the distractor is displayed on the same axis and in the same hemifield as the saccade target. Thus, our prediction was that the eyes would be deviated towards the foveal distractor string, and that the amount of deviation would increase with distractor length since this would shift the centre of gravity towards the fovea.
As illustrated in Figure 1a, there was an overall tendency to undershoot the target letter, and this was greater with longer foveal distractor strings. However, in contradiction with the prediction, the mean landing position error did not vary for distractor strings between 1 and 4-5 letters (about $1.1^{\circ}$ ), and this held for all target eccentricities, while, as shown in Figure 1b, saccade latency tended to increase as the number of letters in the distractor string increased from 1 to 4-5 letters. Thus, short distractor strings failed to deviate the eyes in a centre-of-gravity manner, but tended to produce some sort of a remote distractor effect (see also Benson, this volume). Alternatively, note that short distractor strings favoured the execution of early-triggered small-amplitude saccades that kept the eyes within the distractor string. On that basis, we concluded in favour of a foveal dead zone for the global effect, thus assuming that averaging operates over stimuli displayed outside of a central foveal region of about $1.1^{\circ}$.

Our assumption is that the dead zone is physiologically determined, rather than being central/attentional and/or specific to reading-like situations. First, in our experiments, the target letter, although being most often presented outside of the critical region, was in some cases displayed within this region (e.g. the 3-letter eccentricity condition when target letter strings were 3 letters long; Experiment 3). In those instances, the eyes still accurately landed on target in $42 \%$ of the cases, which suggests that the central foveal region of 4-5 characters was not filtered out by means of attentional processes. In addition, the fact that the eyes sometimes executed early smallamplitude saccades within the dead zone further argues against such an attentional account.

In a more recent study, we attempted to replicate our original findings by manipulating orthogonally the length of the distractor string in number of characters and its extent in degrees of visual angle. As further detailed below, the critical variable appeared to be the angular size of the distractor string, thus indicating, in line with the physiological assumption, that the dead zone did not result from a constant number of letters being filtered out. 


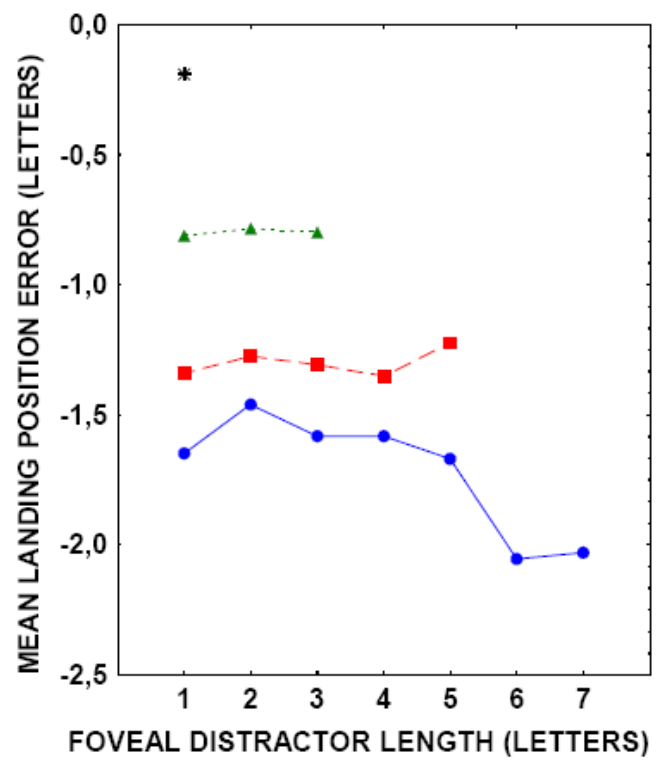

(a)

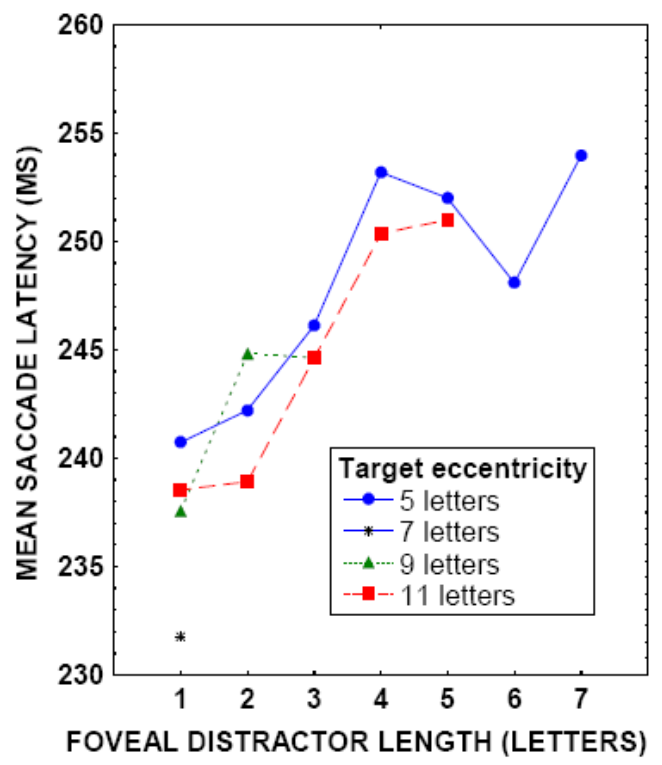

(b)

Figure 1. Re-plotted from Vitu et al.'s (2006) Experiment 1. Mean landing position error in letters (a) and mean latency in ms (b) of the initial saccade made towards the central letter of a 7-letter string (i.e.. 'xxxkxxx') as a function of the length of the foveal distractor string (an x-letter string of a variable number of letters) and the eccentricity of the target letter (5, 7, 9, or 11 letters).

In our character-size experiment ${ }^{1}$, the target, an isolated letter, was displayed in the right hemifield, either in isolation or simultaneously with a distractor string composed of 1 to 7 ' $x$ ' letters; as in our previous studies, the first letter of the distractor was centred on the fixation point and other letters extended further to the right. When initially displayed, the target corresponded to two superimposed letters, ' $h$ ' and ' $k$ '; it was only when a saccade crossed an invisible boundary (located $1^{\circ}$ to the left of the left border of the target letter), that a single letter (' $h$ ' or ' $\mathrm{k}$ ') became visible. In one experimental condition, referred to as the 'small-letter string condition', each letter of the distractor string subtended $.15^{\circ}$ of visual angle, while in the other, the 'large-letter string condition', one letter subtended $.30^{\circ}$ of visual angle (each character space subtended $.22^{\circ}$ and $.42^{\circ}$ respectively); the size of the target letter varied accordingly. The eccentricity of the target letter was maintained constant $\left(3.35^{\circ}\right)$, and hence the distance between the end of the distractor string and the target letter was greater in the small- compared to the large-letter string condition (see Figure 2). Since the deviation of the eyes that results from a global effect depends on the distance between distractor and target, we

1 This experiment was suggested by J. Findlay. added two control conditions with different target eccentricities; in the control small-letter string condition, the eccentricity of the target letter was reduced to $2^{\circ}$ and in the control large-letter string condition, target eccentricity was $4.62^{\circ}$. All conditions were mixed and distributed equally across ten blocks of trials.

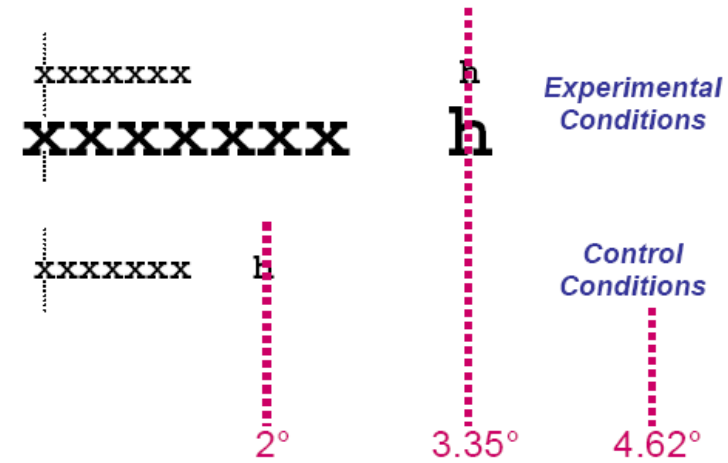

Figure 2. Example stimuli in our character-size experiment. The saccade target stimulus was an isolated letter ( ' $h$ ' in the example) and the distractor was an x-letter string of a variable number of letters. The angular size of the characters was either small $\left(.22^{\circ}\right)$ or large $\left(.42^{\circ}\right)$. In the experimental conditions, the eccentricity of the target letter was maintained constant $\left(3.35^{\circ}\right)$. In the control conditions, it was smaller for small characters $\left(2^{\circ}\right)$, and larger for large characters $\left(4.62^{\circ}\right)$. 
Each trial began with the presentation of two vertically aligned fixation bars; as soon as the computer detected a fixation within less than $.2^{\circ}$ to the left or to the right of the fixation bars, the stimuli were displayed. Eight participants were asked to move their eyes to the target letter as quickly and accurately as possible, while ignoring the distractor string. Their eye movements were recorded with a Dual-Purkinje Image eye tracker, and data were analyzed online with the software developed by van Rensbergen and de Troy (1993; for further details on the procedure and apparatus, see Vitu et al., 2006).

In all conditions, the distributions of initial landing sites were unimodal, the proportion of small-amplitude saccades being much smaller than in our previous study. Figure 3 presents the mean of the initial landing position errors as a function of distractor length and for large- vs.

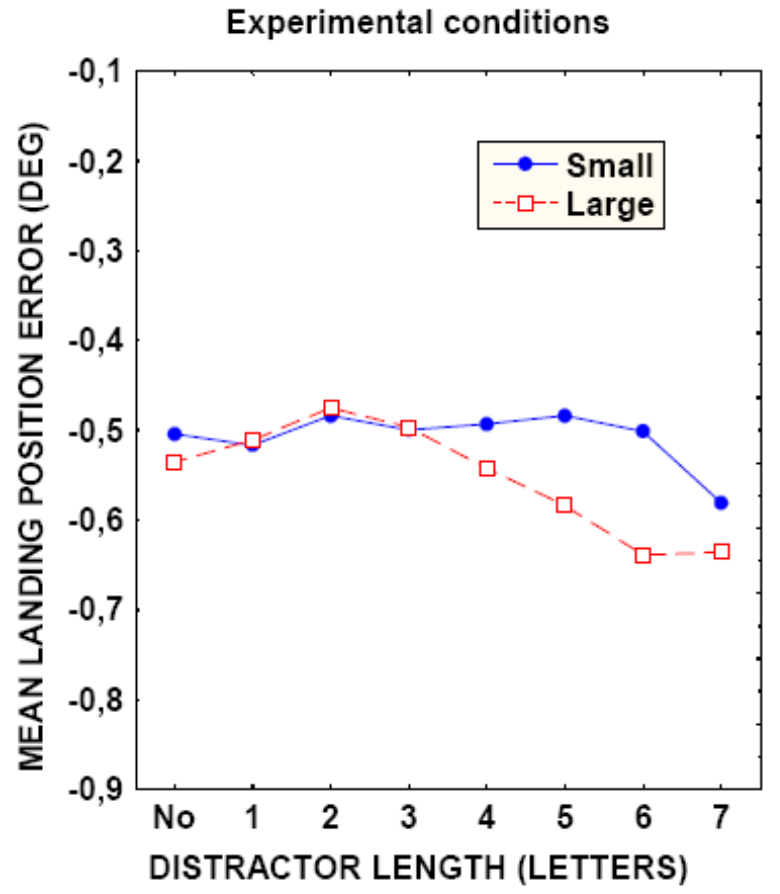

(a) small-letter strings in both the experimental and the control conditions. It clearly shows that an effect of distractor length emerged only for distractor strings that extended further than about $1.2-1.4^{\circ}$ to the right of fixation. In the experimental conditions, there was an overall tendency to undershoot the target letter; the undershoot was of about the same extent in all conditions, but it was greater for distractor strings longer than about 4 letters or $1.4^{\circ}$ in the large-letter string condition, and 6 letters or $1.2^{\circ}$ in the small-letter string condition. The same was true in the two corresponding control conditions, except that the landing position error was overall greater in the largeletter string condition, while it was smaller in the smallletter string condition; this was because the eccentricity of the target letter was respectively greater and smaller than in the experimental conditions.

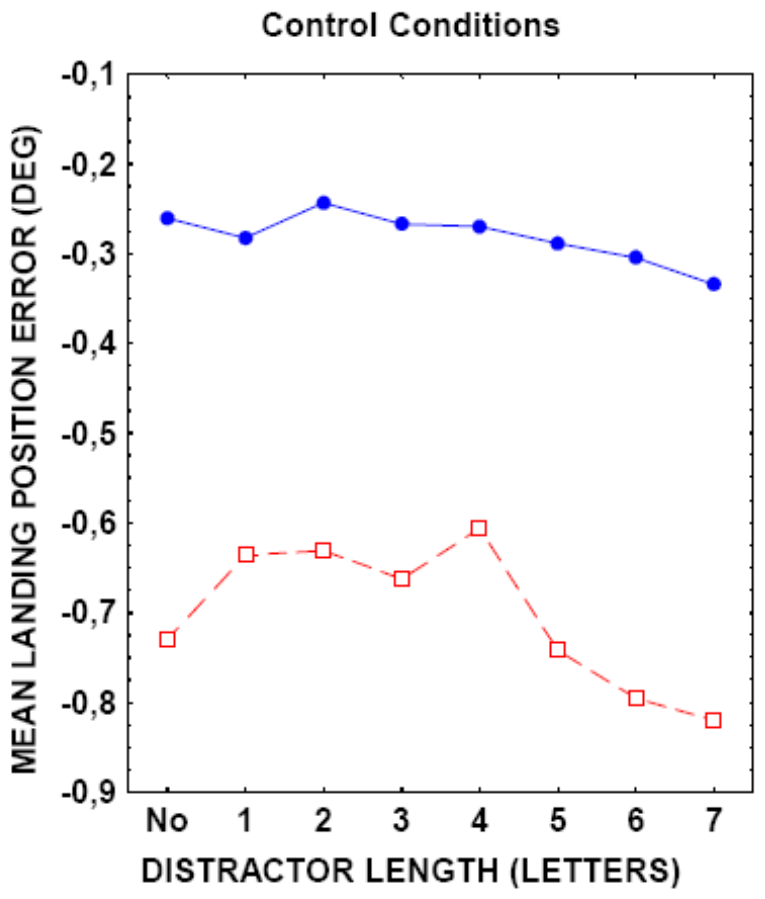

(b)

Figure 3. Mean landing position error (in degrees) of the initial saccade made towards the isolated target letter in the character-size experiment, as a function of the length of the distractor string (in number of letters) and the angular size of the characters (small vs. large) in both the experimental ( $a$ ) and the control ( $b$ ) conditions.

The effects of distractor length and character size were significant as well as the interaction $(\underline{\mathrm{F}}(7,49)=3.67$, $\underline{\mathrm{p}}<.005, \underline{\mathrm{F}}(1,7)=11.92, \underline{\mathrm{p}}<.01$ and $\underline{\mathrm{F}}(7,49)=3.21, \underline{\mathrm{p}}<$ .01 in the experimental condition, and $\underline{F}(7,49)=5.87, \underline{p}<$ $.0005, \underline{F}(1,7)=99.79, \underline{p}<.0005$ and $\underline{F}(7,49)=2.74, \underline{p}<$
.05 in the control condition). For large-letter strings, the linear trend was significant only in the experimental condition $(\underline{F}(1,7)=7.04, \underline{p}<.05$ and $\underline{F}(1,7)=4.18, \underline{p}<.10)$ and the quadratic trend was significant in both the experimental and the control condition $(\underline{F}(1,7)=10.21$, $\underline{p}<$ .01 and $\underline{F}(1,7)=29.26, \underline{p}<.001$ respectively). None of 
these trends were significant in the small-letter string conditions $(\underline{F}(1,7)<=4.18, \underline{p}<.10)$.

As shown in Figure 4, saccade latency was longer when there was a distractor string than when there was not, and this for both character sizes; the effect of distractor length was significant in both the experimental $(\underline{F}(7,49)=10.79, \underline{p}<.0005)$ and the control condition $(\underline{\mathrm{F}}(7,49)=15.47, \underline{\mathrm{p}}<.0005) ;$ this finding is consistent with the previously reported remote distractor effect (Walker et al., 1997; see also Benson, this volume). More surprisingly, saccade latency did not significantly vary for distractor lengths between 1 and 7 letters $(\underline{F}(6,42)=$ .83 and $\underline{F}(6,42)=.61$ in the experimental and control conditions respectively), which is in contrast with some

\section{Experimental conditions}

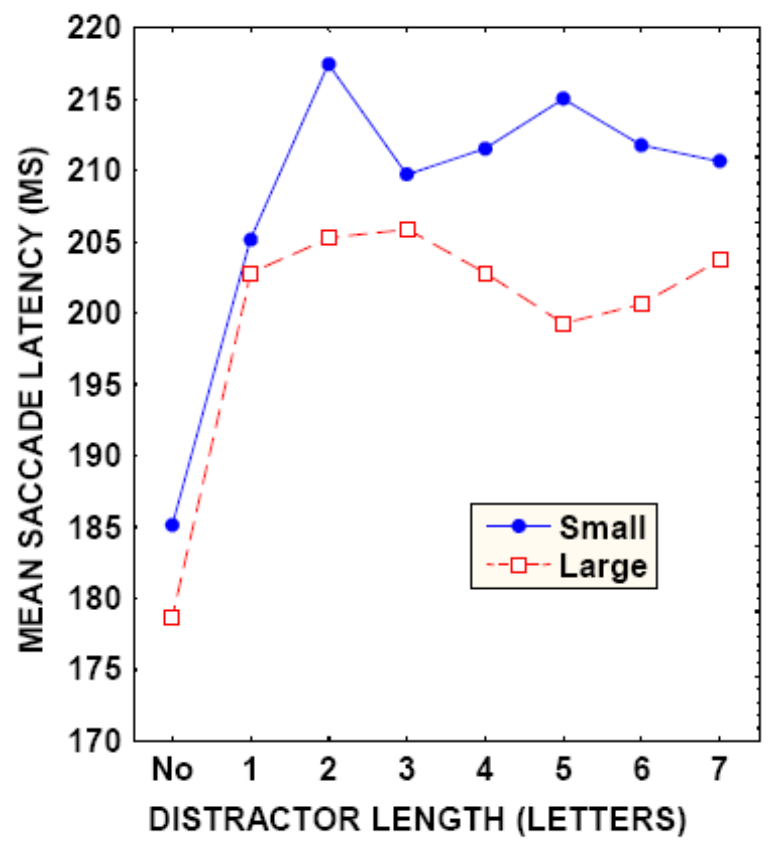

(a) of our previous findings (Vitu et al., 2006; but see Experiment 2 in Vitu et al., 2006) and suggests that saccade latency does not vary systematically with the number of stimuli in the central foveal region. We have at present no explanation for the lack of an effect of distractor length on saccade latencies in the present experiment. However, the simple fact that distractor strings which extended no further than about $1.2-1.4^{\circ}$ from the fixation point failed to deviate the eyes in a centre-of-gravity manner, and this irrespective of character size (or the number of letters), confirms the existence of a dead zone for the global effect, and suggests that the zone is better described in degrees of visual angle than in number of letters.

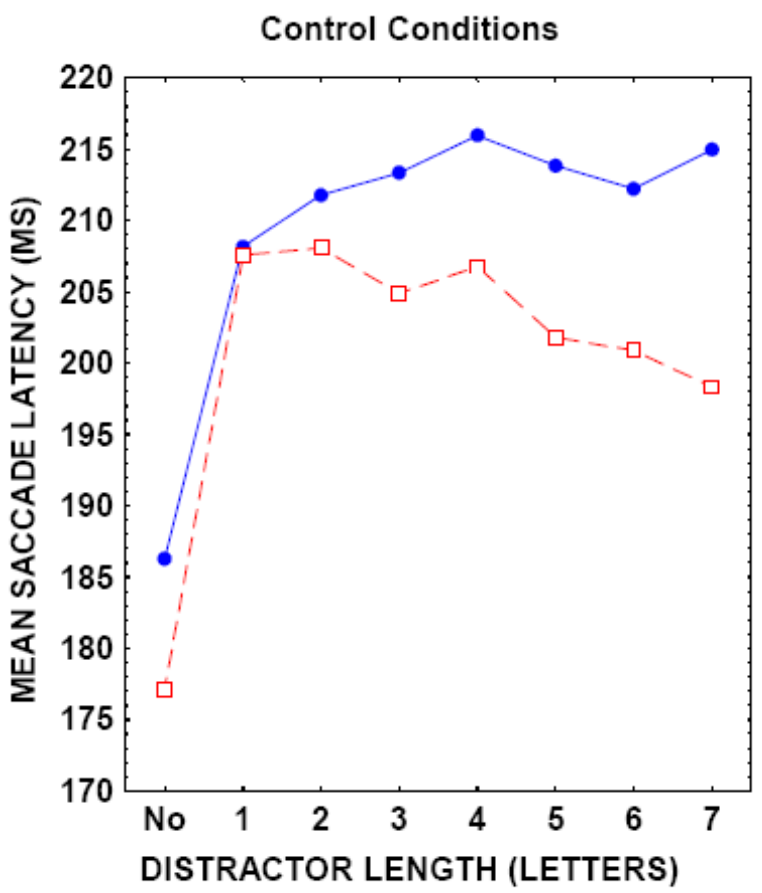

(b)

Figure 4. Mean latency (in ms) of the initial saccade made towards the isolated target letter in the character-size experiment, as a function of the length of the distractor string (in number of letters) and the angular size of the characters (small vs. large) in both the experimental ( $a$ ) and the control (b) conditions.

As we have seen above, contour extraction may contribute to the global effect (Deubel et al., 1988). However, since the ratio of inter-letter spacing to letter size was comparable between small- and large-letter string conditions (.46 vs. .40), it is quite unlikely that differences in terms of contour extraction contributed to the differential effect of distractor length in the two character-size conditions. The lack of a global effect with foveal distractors smaller than $1.2-1.4^{\circ}$ was also not due to distractors being too small to produce a global effect since a global effect was found with peripheral distractor and target stimuli that subtended less than 1 degree of visual angle (e.g. McSorley \& Findlay, 2003). Rather, the critical variable was the part of the retina which the distractor stimulated. Actually, in another recent study, we directly tested the effect of the eccentricity of singleton distractor 
stimuli on the accuracy of saccadic eye movements towards singleton target stimuli; stimuli were either isolated letters or geometrical shapes (Vitu \& Casteau, 2008). In accordance with the foveal dead zone hypothesis, distractors displayed in the same hemifield as the target deviated the eyes in a centre-of-gravity manner, except when they were presented less than about $1^{\circ}$ from the fixation point; in those instances, saccade amplitude remained unaffected, but saccade latency was increased.

We have seen in the first part of the paper that saccade accuracy is a direct function of saccade latency (Viviani \& Swensson, 1982) and that centre-of-gravity trends become less likely as the time for saccade programming increases (Coëffé \& O'Regan, 1987; Vitu et al., 2006). People may argue that failure to observe an influence of distractors displayed within the so-called foveal dead zone came from the fact that in these particular cases, saccade latency was prolonged. In most of the above-mentioned studies, saccade latency and saccade amplitude indeed presented a reciprocal relationship with distractor length or distractor eccentricity. However, this was not always the case; for instance, in our charactersize experiment (see above), distractor strings subtending less than about $1.2-1.4^{\circ}$ failed to produce a global effect, but their latency was not significantly longer than that obtained for larger distractors. Furthermore, as noted by Vitu et al. (2006), averaging responses occur from about $150 \mathrm{~ms}$ from display onset, and such short-latency saccades are also quite frequent in conditions where the distractor fails to produce a global effect. Thus, what we refer to as a foveal dead zone is not the result of a speedaccuracy trade-off.

The exact size of the foveal dead zone remains undetermined at present, but given our complete set of findings, it does not seem to extend further than $1.5^{\circ}$ from the centre of the fovea. It is well known that information within the foveal region is processed differently, and that it is more largely represented than peripheral information (i.e. the cortical magnification factor) at both the level of the visual cortex (Rovamo, Virsu, \& Nasanen, 1978) and the Superior Colliculus (McIlwain, 1991). However, it is quite unlikely that cortical magnification of foveal information is responsible for the dead zone. Indeed, if this were to be the case, then the eyes should land more frequently on the distractor than on the target. However, as we have seen, this behaviour is the exception and not the rule, and again moving the eyes to the peripheral target while a distractor is in the foveal region is not always time consuming, thus suggesting that it does not require the inhibition of a saccade towards the distractor.

A likely interpretation for the foveal dead zone relies on the dissociation between fixation and move systems (Vitu et al., 2006); the former, mainly sensitive to foveal stimulation, would be responsible for keeping the eyes still, while the latter would trigger the execution of saccadic eye movements in response to peripheral stimulation (see Findlay \& Walker, 1999; Walker et al., 1997). As suggested by several authors, this dissociation may be present at the level of the Superior Colliculus where two distinct populations of neurons have been identified $(\mathrm{Mu}-$ noz \& Wurtz, 1993a, 1993b; but see Goffart, Hafed, Dill, \& Krauzlis, 2006). Fixation neurons discharge during fixation, while they remain silent during saccade execution; their stimulation either delays saccade onset or triggers the execution of small amplitude saccades (Munoz \& Fecteau, 2002). They are mainly located in the rostral pole region of the Superior Colliculus, which receives input from the 2-degree foveal region of the retina $(\mathrm{Mu}-$ noz \& Wurtz, 1993a, 1993b), though they might also be present in smaller proportions up to about $10^{\circ}$ from the centre of the fovea (Gandhi \& Keller, 1999). Saccadic neurons are in contrast associated with peripheral information and they discharge before and during saccade execution.

Walker et al. (1997) accounted for the remote distractor effect based on the dissociation between fixation and move neurons. A similar interpretation may also hold for the foveal dead zone (Vitu et al., 2006). When two stimuli are simultaneously displayed in the same hemifield, but one falls in the central foveal region, averaging fails to occur because two functionally different populations of neurons, or two different subsystems with different time courses, are activated. Future studies will further investigate the neural basis of the foveal dead zone and the related remote distractor effect, as recent neurophysiological findings challenge the existence of fixation neurons at the level of the Superior Colliculus (Goffart et al., 2006). However, as we have seen, it is quite clear that these phenomena are not experimental artefacts, but result from the properties of the oculomotor system. 
The global effect within a limited peripheral window

As we have just seen, when distractor and target stimuli are displayed in the same hemifield along the horizontal axis, only the distractors outside of a foveal dead zone of about $1-1.5^{\circ}$ deviate the eyes in a centre-ofgravity manner. As suggested by a large number of studies, it is quite unlikely that all elements outside of the foveal dead zone contribute to the same extent to computation of saccade amplitude. Findlay (1982) was the first to note that the eyes are more largely deviated towards the stimuli that are closer to the fovea. This observation was replicated in several other studies, and the proposal was made that this is due to the non-homogeneity of retinal projections (Coëffé \& O'Regan, 1987; Findlay, Brown, \& Glichrist, 2001; Vitu, 1991a; but see McGowan et al., 1998). We mentioned above that it is quite unlikely that cortical magnification of foveal information is responsible for the foveal dead zone. However, this probably plays a role in determining the respective contributions of more eccentric visual elements, even though as we will see, this is probably not the only variable involved.

In an earlier study, we tested the influence of distractor stimuli (random dot patterns) on the distribution of initial landing sites in saccade target words (Vitu, 1991a). Target words were either 5 or 9 letters long; they were displayed simultaneously with a word of the same length to their right. Distractors were presented at four different locations above and below the target word: to the left of the beginning and of the centre of the word and to the right of the centre and of the end of the word (see Figure 5).

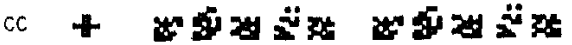

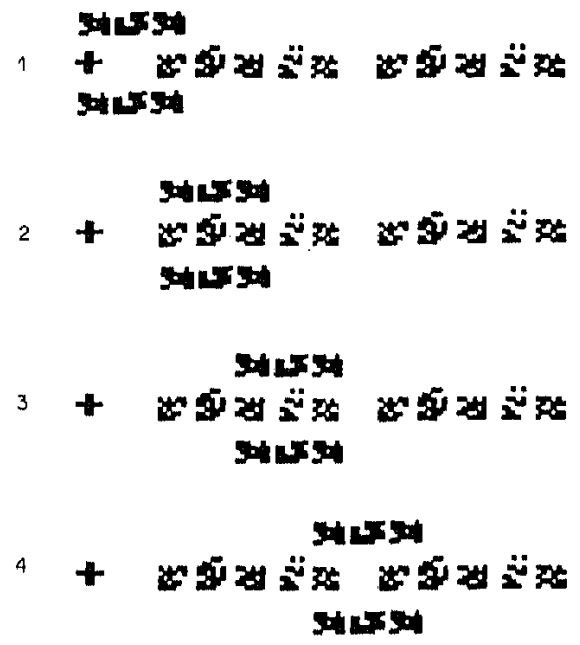

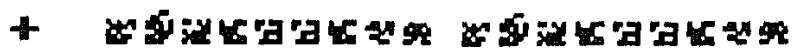

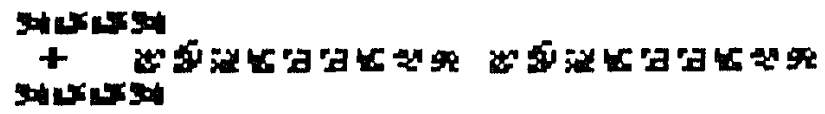

\section{3atroryd

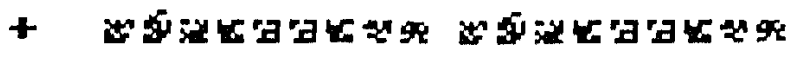

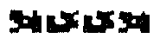

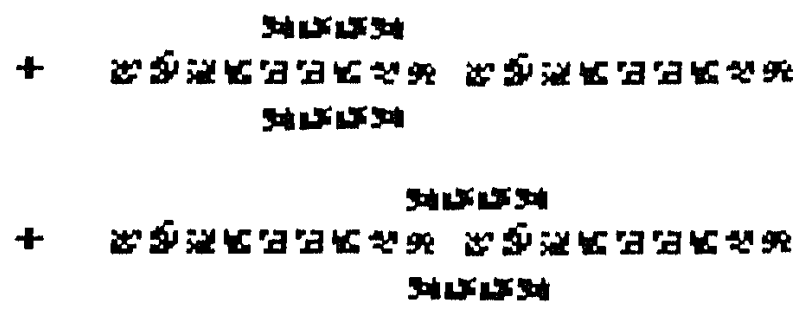

Figure 5. Example stimuli in Vitu's (1991) Experiment 3. The target stimulus was a word of 5 or 9 letters. This was presented simultaneously with another word to its right (in the illustration, the words were masked in peripheral vision, and they became visible only when a saccade towards the first word was detected) and distractor stimuli (random dots patterns equivalent to masked letters). Distractors were displayed to the left of the beginning or of the centre of the first word, or to the right of the word's centre or end (Positions 1-4). In a control condition (CC), the two words were displayed with no distractor stimulus.

Results showed that leftward-displayed distractors did not significantly deviate the eyes as compared to a control condition with no distractor; the eyes landed on average near the centre of the word or slightly left of it. Note that when the distractors were displayed in front of the word's beginning, they fall in a $1.2^{\circ}$ foveal region (i.e. the foveal dead zone; see above), which explains why they had no effect. In contrast, in the two conditions where the distractors were presented to the right of the word's centre, the distribution was significantly shifted 
towards the end of the word. Interestingly, the deviation increased with distractor eccentricity for 5- but not 9letter words, thus suggesting that distractors that were too eccentric did not contribute to computation of saccade amplitude.

Implementation of different centre-of-gravity assumptions provided further evidence for a limited influence of distant distractors. Figure 6 shows that the geometrical centre of gravity of the overall configuration formed by the letters of the two presented words and the distractor patterns largely overestimated the eyes' landing position in 5- and 9-letter words. The cortically-weighted centre of gravity of the global configuration (using a cortical magnification factor of 1.7 as suggested by O'Regan, 1989, 1990) gave a slightly better fit, but again overestimated the mean landing position. The best fit was actu- ally obtained when the cortically-weighted centre of gravity of a subset of the visual elements in the periphery, i.e. the elements contained in a 7-letter (or $4.2^{\circ}$ ) window from the beginning of the target word, was computed. The window thus excluded (1) the distractor stimuli when they were located in front of the target word or in the foveal dead zone, but also (2) the elements that were further than 7-letters (or $4.2^{\circ}$ ) from the beginning of the target word. These data suggest that the stimuli that were too eccentric did not contribute at all and hence that the centre of gravity was computed within a limited peripheral window rather than being based on the full peripheral configuration. The cortical magnification factor slightly modulated the respective contribution of the visual elements in the critical window, but could not be directly responsible for the abrupt peripheral cut-off.

\section{5-letter words (Vitu, 1991a -Experiment 3)}

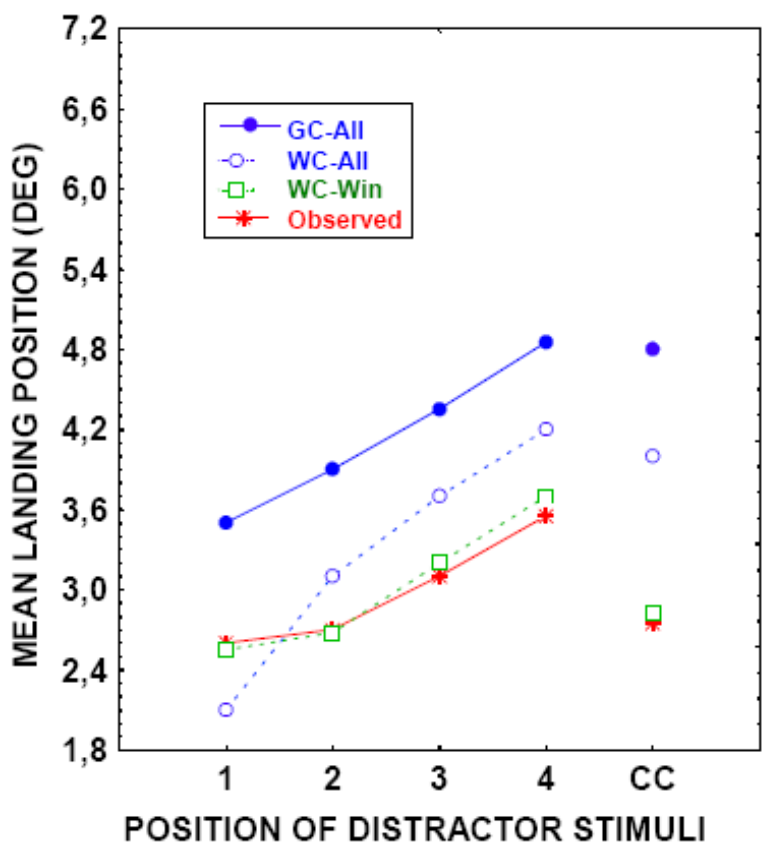

(a)

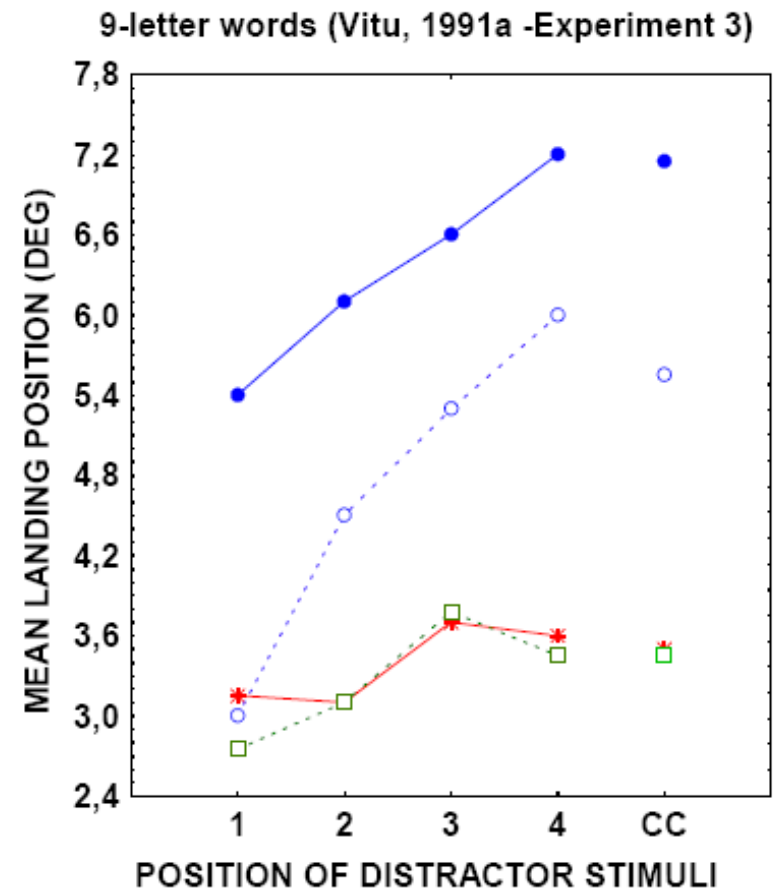

(b)

Figure 6. Re-plotted from Vitu's (1991) Experiment 3. Observed and predicted mean initial landing positions in the first word (in degrees) as a function of the position of the distractor pattern (1-4) and in the control condition (CC) for 5-and 9-letter words (a-b respectively). Predictions were derived from alternative versions of the centre-of-gravity assumption: the geometrical and the cortically-weighted centre of gravity of the full configuration (including all stimuli to the right of the fixation point; GC-all and WCall respectively), and the cortically-weighted centre of gravity of a 7-letter (or $\left.4.2^{\circ}\right)$ peripheral window from the beginning of the first word (or $1.2^{\circ}$ from the fixation point; WC-win respectively). The angular position of the cortically-weighted centre of gravity (CW) was calculated using the following formula, $\sum[\Phi i /(1+1.7 \Phi i)]=n * C W /(1+1.7 * C W)$, where $\Phi i$ is the eccentricity of each visual element (letter or random dot pattern) in the configuration, and $n$ the total number of elements in the configuration. 
Vitu et al.'s (2006) recent findings further support the hypothesis that the global effect operates within a limited peripheral window. Recall that in the experiments, participants were simultaneously presented with four letter strings in the right hemifield: a distractor string of variable length whose first letter was centred on the initial fixation point, two target-letter strings of same lengths (e.g. 'xxxkxxx'), and an additional 9-letter string. The initial target letter (the centre of the initial target string) was presented at variable eccentricities, from 5 to 11 letters (or 1.55 to 3.41 degrees), which progressively shifted the whole configuration (except the distractor string) further towards the periphery. In Figure 7, we re-plotted the mean landing position error of the initial saccades in the 1-letter distractor condition of Vitu et al.'s Experiment 1 as a function of target eccentricity, and contrasted the obtained curve with the predictions made by alternative versions of the centre-of-gravity hypothesis.

\section{Vitu et al. (2006) -Experiment 1}

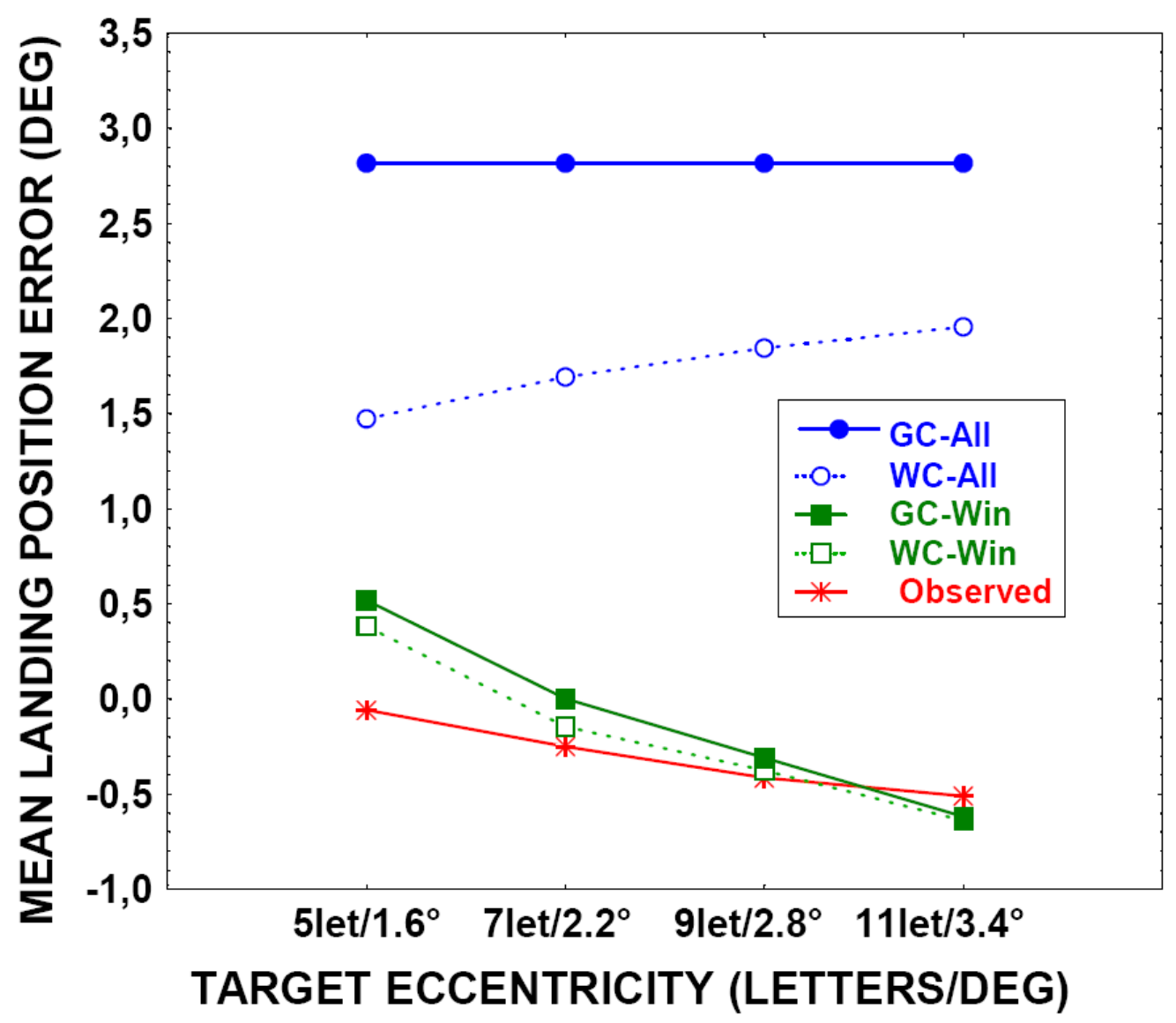

Figure 7. The observed and predicted mean landing position error of the initial saccade as a function of the eccentricity of the target letter (5-11 letters or 1.6-3.4 ) in Vitu et al.'s (2006) Experiment 1; the target letter was embedded in a 7-letter string presented simultaneously with a single distractor letter at fixation, and two peripheral letter strings (of 7 and 9 letters respectively) to its right. Predictions were derived from alternative versions of the centre-of-gravity assumption: the geometrical and the cortically-weighted centre of gravity of the full configuration (including all stimuli to the right of the fixation point; GC-all and WC-all respectively), and the geometrical and the cortically-weighted centre of gravity of a peripheral window of $2.4^{\circ}$ from the end of the foveal dead zone (or $1.1^{\circ}$ from the fixation point; $G C$-win and $W C$-win respectively). The angular position of the cortically-weighted centre of gravity $(C W)$ was calculated using the same formula as in Figure 6. 
Both the geometrical and the weighted centre of gravity of the global configuration formed by all letters to the right of fixation largely overestimated the mean initial landing position, and both failed to reproduce the effect of target eccentricity or the fact that target letters were more largely undershot as their eccentricity increased; note that the weighted centre of gravity of the configuration formed by a constant number of letters from the beginning of the first target letter string (not shown here) also failed to reproduce the effect of target eccentricity. However, when the centre of gravity was computed over the subset of letters contained in a peripheral window of $2.4^{\circ}$ from the end of the estimated foveal dead zone (i.e. $1.1^{\circ}$ to the right of fixation), the fit was greatly improved, being only slightly better for the weighted compared to the geometrical centre of gravity. In addition, an effect of target eccentricity in the same direction as the observed effect was this time predicted, thus confirming that only a subset of the letters in the periphery contributed to computation of saccade amplitude.

Still, the slope of the estimated curve was slightly greater than the slope of the observed curve, and the fit was particularly poor for the 5-letter eccentricity condition. As we noted above, the exact size of the foveal dead zone remains undetermined, and it may well be that the zone is not sharp-edged, but defined by a gradient. This may be one of the reasons why the fit was rather poor in the 5-letter eccentricity condition. Since the first letters of the initial letter string fall into the $1.1^{\circ}$ foveal dead zone, the centre of gravity was computed over the remaining set of letters in the string. Thus, overestimation of the eyes' landing position could be due to a too large number of letters being filtered out in this condition. In addition, as further detailed below, the size of the peripheral window is probably not fixed; it varies over time and as a function of the eccentricity of the stimulus pattern. This may also explain why our predictions did not perfectly fit the observed data.

We initially interpreted the critical peripheral window in terms of selective attentional processes, thus assuming that the eyes first move towards the weighted centre of gravity of a subset of selected peripheral stimuli (Vitu, 1991a; see also Coëffé \& O'Regan, 1987; Findlay \& Kapoula, 1992; Findlay \& Walker, 1999). Such attentional processes may serve to avoid the influence of too distant elements while taking the eyes closer to the saccade target location (Findlay \& Kapoula, 1992; Vitu, 1991a).
Alternatively, as we recently proposed, the null or reduced contribution of stimuli outside of a critical peripheral window may simply arise from the patterns of activity in the saliency map that result from multiple stimulations (Vitu et al., 2006). Elements that are too eccentric would not be integrated simply because they generate activity patterns that are too weak relative to the main peak of activity ${ }^{2}$. Since elements that are closer to the fovea presumably produce stronger signals (i.e. the cortical magnification factor), they probably form a first main peak of activity, which naturally filters out elements that are too distant and that produce comparatively much weaker signals. As the first main peak of activity (or the stimulus configuration) shifts further towards the periphery, it becomes weaker, which may in turn increase the contribution of more distant elements. Note however that in the end, the eyes more greatly undershoot the saccade target location as the eccentricity of the stimulus pattern increases (see Figure 7) because elements that are more eccentric than the saccade target are less and less likely to be integrated to the main peak of activity; even if the angular extent of the window from the end of the foveal dead zone slightly increases with eccentricity, the number of visual stimuli contained in the window decreases.

The size of the critical peripheral window may also vary over time. The saliency landscape greatly changes with saccade latency, and as time goes by attentional selection processes are more likely to intervene; these strongly reduce the extent of the critical peripheral window, and hence the deviating power of distractor elements. As we previously noted, the time course of selective processes varies with the eccentricity of the saccade target, being faster for closest and hence most visible targets (Vitu et al., 2006). This suggests that time-related changes in the patterns of activity may also contribute to the effect of the eccentricity of the stimulus pattern on initial saccades' landing positions.

The model proposed above is obviously too simplistic and unable to account for our complete set of findings, and further developments will be necessary. However, it seems reasonable to conclude at this stage that computation of saccade amplitude in response to multiple stimula-

\footnotetext{
2 The fact that the estimated size of the peripheral window in Vitu's (1991) study was about twice as large as the one estimated based on Vitu et al. 's (2006) findings, i.e. $4.2^{\circ} \mathrm{vs.} 2.4^{\circ}$, is actually consistent with this assumption since characters were about twice as large in the former case.
} 
tion results from the interplay of several parameters including the spatial arrangement of the stimuli, retinal eccentricity and saccade latency. These parameters affect the size of the critical peripheral window over which visual integration processes operate; they also affect to a certain extent the respective contributions of the visual elements within the critical region.

\section{Accounting for eye movements in reading}

Reading is a particular and complex perceptual task. It involves a large range of processes such as feature extraction, letter identification, lexical access and higher-level syntactic and semantic integration processes. However, from an oculomotor point of view, this may be considered as a simple task which basically consists of making series of horizontal saccades in response to multiple foveal and peripheral stimulations, and in that sense it may be the ideal situation for the global effect to express itself. The observation we made several years ago, and which has been replicated since then, that eye movements in 'z-reading' or during the scanning of meaningless $\mathrm{z}$ letter strings closely resemble the eye movement pattern that is typical of normal reading corroborates this view (Nuthmann, Engbert, \& Kliegl, 2007; Rayner \& Fischer, 1996; Vitu, O'Regan, Inhoff, \& Topolski, 1995; see also Nazir, 1991) ${ }^{3}$. Indeed, this suggests that eye movements in reading are mainly determined by low-level visuomotor processes; ongoing word identification processes would only intervene occasionally to modulate the default eye movement pattern (see Vitu, 2003). This, together with the fact, as we have seen above, that a global effect was found with reading like material and isolated words suggests that global visual integration processes may greatly contribute to eye guidance in reading.

Our assumption is that the eyes move forward along the lines of text in a rather unintelligent manner, as if they were pulled by the visual material ahead of fixation (Vitu,

3 Note that Rayner and Fischer (1996) mainly discussed the dissimilarities between text reading and z-reading, and on that basis, concluded that cognitive processes are the main determinant of eye behaviour. However, their data were very similar to ours since they replicated very robust phenomena with z-letter string materials such as the relationship between word skipping and word length (i.e. the fact that words become less likely to be skipped as their length increases) and the Preferred Viewing Position effect (i.e. the general tendency to fixate the centre of words or slightly left of it).
2003; Vitu et al., 2006). The words and letters that compose a line of text generate activity patterns within a visually-defined saliency map; these form the basis for determining saccade amplitude. Each time the eyes move, they move towards the point of maximum visual saliency at the time the saccade starts being computed (see also Findlay \& Walker, 1999). As we have seen above, visual saliency is not only determined by the visual properties of the stimuli, but it depends also strongly on global visual integration processes, suggesting therefore that the eyes are sent towards the weighted centre of gravity of the configuration formed by the words to the right of fixation. Thus, what mainly matters is not the meaning of the words or their lexical properties, but rather the words' spatial arrangements and their lengths, which word(s) and letters fall within critical regions such as the foveal dead zone and the critical peripheral window, and time required for the saccade to be computed. The latter variable is quite critical since the saliency map shows dynamical changes over time and since fixation durations in reading show great variability ${ }^{4}$.

According to our view, visual selection processes are the exception and not the rule; since they take time to emerge, they can only intervene occasionally to determine saccade amplitude. Thus, rather than being sent to a specific target location in the periphery, the eyes are simply sent forward towards the point of maximum saliency. This point is probably what makes our theory so different from other proposals. Indeed, in most theories and models of eye-movement control in reading, it is classically assumed that the eyes aim for the centre of peripherally selected target words, with words being selected most often based on ongoing processes (Engbert, Nuthmann, Richter, \& Kliegl, 2005; Just \& Carpenter, 1980; McDonald, Carpenter, \& Shillcock, 2005; O'Regan, 1990, 1992; O'Regan \& Lévy-Schoen, 1987; Reichle, Rayner, \& Pollatsek, 2003; Reilly \& Radach, 2003, 2006; Reilly \& O'Regan, 1998; but see Yang, 2006; Yang \& McConkie, 2001, 2004). As we noted in several review papers, the likelihood of skipping a word is mainly a function of the length of the word and the saccade launch distance with respect to the beginning of the word (see below), and it is very rarely and only slightly affected by

\footnotetext{
4 The processes determining fixation durations in reading are not described in the present paper, but were discussed in a recently published paper (Vitu, Lancelin \& Marrier d'Unienville, 2007).
} 
linguistic variables (Brysbaert, Drieghe, \& Vitu, 2005; Brysbaert \& Vitu, 1998). This confirms a minor role of language processes and suggests that selective eye guidance based on parafoveal word identification processes can intervene only occasionally ${ }^{5}$. Furthermore, two main arguments can be raised against the classical assumption that the eyes aim for the centre of peripherally selected target words. The first argument relates to saccade accuracy with complex stimulus patterns. As we have seen above, the accuracy of saccadic eye movements to the central target letter of a peripheral letter string is very poor, and it is only when saccade latency is in the order of $300-600 \mathrm{~ms}$, that the eyes accurately land on target (Vitu et al., 2006; see also Coëffé \& O'Regan, 1987; Jacobs, 1987). Since fixation durations in reading are, on average, about $225 \mathrm{~ms}$, it seems quite unlikely that the function of saccades is to send the eyes to a specific location in a peripherally defined target word. If it were so, this would mean that the eyes consistently attempt to do something that they frequently fail to achieve! The second argument was given earlier in the manuscript. We showed, based on two data sets collected respectively during the reading of isolated words and in saccade target tasks involving reading-like material, that the mean initial landing position in the configuration could be predicted by simply computing a weighted centre of gravity of the stimuli displayed in a critical peripheral window. The contribution of each element in the window was weighted by its eccentricity, but no particular weight was attributed to a given word/string or to the central letter of the words/strings or any other assumed target location. This suggests that the notion of saccade target is not necessary to account for initial landing sites in words. In a more general manner, there may be no need to consider word skip cases as specific instances that result from selectiontype processes. Indeed, centre-of-gravity type processes can lead the eyes to skip words, particularly short words.

Another critical difference of our theory with previous views relates to the major role given to global visual integration processes. These have been rarely considered as being part of the visuo-motor processes underlying eye guidance in reading. One of the only exceptions was O'Regan and Lévy-Schoen's (1987) strategy-tactics theory (see also O'Regan, 1990, 1992), and here, global vis-

5 This may only apply to progressive saccades but not regressions, although the determinants of regressive saccades will not be discussed in the present paper (for a review see Vitu, 2005). ual integration processes were not the main driving force; they were only responsible for oculomotor aiming errors that prevented the eyes attaining the aimed-for location (i.e. the centre of words). In all current models of eyemovement control in reading, global visual integration processes are completely absent; the basic assumption is that the eyes aim for the centre of words, and that the variability of initial landing sites results from systematic oculomotor range error (Engbert et al., 2005; McDonald et al., 2005; Reichle et al., 2003). Actually, this proposal was initially made by McConkie et al. (1988) who showed that the distribution of initial landing sites in words, a Gaussian centred on the word's centre or slightly left of it (i.e. the Preferred Viewing Position effect; Rayner, 1979), is the sum of individual Gaussian distributions associated with different saccade launch sites. The launch site is the distance of the eyes to the beginning of a word before the saccade is executed. This affects the eyes' landing position in words in a quite typical manner: the eyes overshoot the centre of a word when they are launched from close to the beginning of the word, while they undershoot the centre of words when they are launched from further away. The similarity of this phenomenon with the range effect, or the tendency to send the eyes towards the centre of the range of target eccentricities in a block of trials (Kapoula, 1985) led the authors to propose that the eyes are sent towards the centre of words (or their corresponding visual blobs), and that the variability of landing sites around this preferred location results from a bias towards executing saccades of a constant length (i.e. the average saccade length in the task).

We previously challenged the systematic range error assumption; in one of our early experiments, we manipulated the eccentricity of pairs of isolated words within blocks of trials, and we failed to find significant shifts of the distributions of initial landing sites in words as a function of the eccentricity of the words (Vitu, 1991b). More recently, we reported that the eccentricity of targetletter strings influences the distribution of initial landing sites, but our pattern of findings did not resemble a range effect; far targets were undershot, but close targets were not systematically overshot (Vitu et al., 2006; see also Coëffé \& O'Regan, 1987). Furthermore, the eccentricity effect could be reproduced by computing the weighted centre of gravity of the set of letters contained in a limited peripheral window (see Figure 7). Even though the predictions of our model did not perfectly fit the observed 
data, the simple fact that an effect of eccentricity in the correct direction could be predicted based on a centre-ofgravity assumption, shows that the launch site effect could well result from global integration processes instead of systematic oculomotor range error. In reading, the eyes tend to overshoot the centre of words (and to skip words) when they are launched from close to the beginning of words because they are pulled forward by letters to the right of the word's centre, eventually including those belonging to one of the following words; in contrast, the eyes undershoot the centre of words when they are launched from further locations, because intermediate letters between the end of the foveal dead zone and the centre of the word retain the eyes from moving too far. Note that in line with the present assumption, we simply failed to replicate Kapoula's (1985) original findings with singleton target stimuli (Nuthmann, Vitu, Kliegl, \& Engbert, in preparation; see also Findlay, 1982); these data challenge the existence itself of a range effect in the oculomotor system.

Of course, the present assumptions will need to be implemented in a more complete model and be further validated. However, as we have seen here, the global effect and the related centre-of-gravity idea are key notions that could explain the placement of the eyes along the lines of text in reading and that certainly explain more data than those collected in simple oculomotor tasks.

\section{Acknowledgments}

This work greatly benefited from interactions with John Findlay over the past 20 years, whom I would like to warmly thank. I would also like to greatly thank R. Engbert, R. Kliegl, A. Nuthmann and other members of the Potsdam group for their encouragements and very helpful feedback during my stay at the University of Potsdam during the fall 2005. I am also grateful to A. Lévy-Schoen and J. K. O'Regan who gave me the tools to develop these ideas. The present work also benefited from presentation and interactions at ECEM $14^{\text {th }}$ in Potsdam and at the 'Journée Oculomotricité' organized by the 'GDR Vision' in Lille, France, in January 2008. H. Blythe and S.P. Liversedge read the first version of this manuscript, and I would like to thank them for their very helpful comments.

\section{References}

Arai, K., McPeek, R. M., \& Keller, E. L. (2004). Properties of saccadic responses in monkey when multiple competing visual stimuli are present. Journal of Neurophysiology, 91, 890-900.

Brysbaert, M., Drieghe, D., \& Vitu, F. (2005). Word skipping: Implications for theories of eye movement control in reading. In G. Underwood (Ed.), Cognitive processes in eye guidance. (pp. 53-77). Oxford, NY: Oxford University Press.

Brysbaert, M., \& Vitu, F. (1998). Word skipping: Implications for theories of eye movement control in reading. In G. Underwood (Ed.), Eye guidance in reading and scene perception. (pp. 125-147). Oxford: Elsevier.

Chou, I. H., Sommer, M. A., \& Schiller, P. H. (1999). Express averaging saccades in monkeys. Vision Research, 39(25), 4200-4216.

Coëffé, C., \& O'Regan, J. K. (1987). Reducing the influence of non-target stimuli on saccade accuracy: Predictability and latency effects. Vision Research, 27, 227-240.

Cohen, M. E., \& Ross, L. E. (1978). Latency and accuracy characteristics of saccades and corrective saccades in children and adults. Journal of Experimental Child Psychology, 26, 517-527.

Coren, S., \& Hoenig, P. (1972). Effects of non-target stimuli upon length of volontary saccades. Perceptual and Motor Skills, 34, 499-508.

Deubel, H., Findlay, J. M., Jacobs, A., \& Brogan. (1988). Saccadic eye movements to targets defined by structure differences. In G. Lüer, U. Lass \& J. ShalloHoffmann (Eds.), Eye movement research: Physiological and psychological aspects (pp. 107-145). Toronto: Hogrefe.

Deubel, H., Wolf, W., \& Hauske, G. (1984). The evaluation of the oculomotor error signal. In A. G. Gale \& F. Johnson (Eds.), Theoretical and applied aspects of eye movement research (pp. 55-62). Elsevier Science Publishers B.V. (North Holland).

Edelman, J. A., \& Keller, E. L. (1998). Dependence on target configuration of express saccade-related activity in the primate superior colliculus. Journal of Neurophysiology, 80(3), 1407-1426. 
Eggert, T., Sailer, U., Ditterich, J., \& Straube, A. (2002). Differential effect of a distractor on primary saccades and perceptual localization. Vision Research, 42, 2969-2984.

Engbert, R., Nuthmann, A., Richter, E., \& Kliegl, R. (2005). Swift: A dynamical model of saccade generation during reading. Psychological Review, 112(4), 777-813.

Findlay, J. M. (1982). Global visual processing for saccadic eye movements. Vision Research, 22, 10331045.

Findlay, J. M. (1997). Saccade target selection during visual search. Vision Research, 37(5), 617-631.

Findlay, J. M. (2004). Eye scanning and visual search. In F. Ferreira \& J. Henderson (Eds.), Interface of language, vision, and action: Eye movements and the visual world (pp. 135-159). New York: Psychology Press.

Findlay, J. M., \& Blythe, H. I. (in press). Saccade target selection: Do distractors affect saccade accuracy? Vision Research.

Findlay, J. M., Brogan, D., \& Wenban-Smith, M. G. (1993). The spatial signal for saccadic eye movements emphasizes visual boundaries. Perception \& Psychophysics, 53(6), 633-641.

Findlay, J. M., \& Brown, V. (2006). Eye scanning of multi-element displays: Ii. Saccade planning. Vision Research, 46(1-2), 216-227.

Findlay, J. M., Brown, V., \& Glichrist, I. D. (2001). Saccade target selection in visual search: The effect of information from the previous fixation. Vision Research, 41(1), 87-95.

Findlay, J. M., \& Gilchrist, I. D. (1997). Spatial scale and saccade programming. Perception, 26, 1159-1167.

Findlay, J. M., \& Kapoula, Z. (1992). Scrutinization, spatial attention, and the spatial programming of saccadic eye movements. The Quarterly Journal of Experimental Psychology, 45A(4), 633-647.

Findlay, J. M., \& Walker, R. (1999). A model of saccade generation based on parallel processing and competitive inhibition. Behavioral and Brain Sciences, 22(4), 661-720.
Gandhi, N. J., \& Keller, E. L. (1999). Comparison of saccades perturbed by stimulation of the rostral superior colliculus, the caudal superior colliculus, and the omnipause neuron region. Journal of Neurophysiology, 82(6), 3236-3253.

Godjin, R., \& Theeuwes, J. (2002). Programming of endogenous and exogenous saccades: Evidence for a competitive integration model. Journal of Experimental Psychology:Human Perception and Performance, 28(5), 1039-1054.

Goffart, L., Hafed, Z. M., Dill, N., \& Krauzlis, R. J. (2006). Changes in eye position during fixation caused by inactivation of the rostral superior colliculus. Neuroscience.

Guez, J. E., Marchal, P., Le Gargasson, J. F., Grall, Y., \& O'Regan, J. K. (1994). Eye fixations near corners: Evidence for a centre of gravity calculation based on contrast, rather than luminance or curvature. Vision Research, 34(12), 1625-1635.

He, P. Y., \& Kowler, E. (1989). The role of location probability in the programming of saccades: Implications for "center-of-gravity" tendencies. Vision Research, 29(9), 1165-1181.

He, P. Y., \& Kowler, E. (1991). Saccadic localization of eccentric forms. Journal of the Optical Society of America, 8(2), 440-449.

Jacobs, A. (1987). On localization and saccade programming. Vision Research, 27(11), 1953-1966.

Just, M. A., \& Carpenter, P. A. (1980). A theory of reading: From eye fixations to comprehension. Psychological Review, 87, 329-354.

Kapoula, Z. (1985). Evidence for a range effect in the saccadic system. Vision Research, 25(8), 1155-1157.

Lee, C., Rohrer, W. H., \& Sparks, D. L. (1988). Population coding of saccadic eye movements by neurons in the superior colliculus. Nature, 332(6162), 357-360.

McConkie, G. W., Kerr, P. W., Reddix, M. D., \& Zola, D. (1988). Eye movement control during reading: I. The location of initial eye fixations on words. Vision Research, 28(10), 1107-1118.

McDonald, S. A., Carpenter, R. H. S., \& Shillcock, R. C. (2005). An anatomically constrained, stochastic model of eye movement control in reading. Psychological Review, 112, 814-840. 
McGowan, J. W., Kowler, E., Sharma, A., \& Chubb, C. (1998). Saccadic localization of random dot targets. Vision Research, 38(6), 895-909.

McIlwain, J. T. (1976). Large receptive fields and spatial transformations in the visual system. In R. Porter (Ed.), International review of physiology, vol 10, neurophysiology ii (pp. 223-247).

McIlwain, J. T. (1991). Distributed spatial coding in the superior colliculus. Visual Neuroscience, 6, 3-13.

McSorley, E., \& Findlay, J. M. (2003). Saccade target selection in visual search: Accuracy improves when more distractors are present. Journal of Vision, 3, 877-892.

Melcher, D., \& Kowler, E. (2001). Visual scene memory and the guidance of saccadic eye movements. Vision Research, 41, 3597-3611.

Menz, C., \& Gröner, R. (1987). Saccadic programming with multiple targets under different task conditions. In J. K. O'Regan \& A. Lévy-Schoen (Eds.), Eye movements: From physiology to cognition. Amsterdam: North-Holland.

Munoz, D. P., \& Fecteau, J. H. (2002). Vying for dominance: Dynamic interactions control visual fixation and saccadic initiation in the superior colliculus. In J. Hyönä, D. P. Munoz, W. Heide \& R. Radach (Eds.), Progress in brain research (Vol. 140, pp. 3-19): Elsevier Science B.V.

Munoz, D. P., \& Wurtz, R. H. (1993a). Fixation cells in monkey superior colliculus. I. Characteristics of cell discharge. Journal of Neurophysiology, 70(2), 559575.

Munoz, D. P., \& Wurtz, R. H. (1993b). Fixation cells in monkey superior colliculus. Ii. Reversible activation and deactivation. Journal of Neurophysiology, 70(2), 576-589.

Nazir, T. (1991). On the role of refixations in letter strings: The influence of oculomotor factors. Perception and Psychophysics, 49(4), 373-389.

Nuthmann, A., Engbert, R., \& Kliegl, R. (2007). The iovp effect in mindless reading: Experiment and modeling. Vision Research, 47, 990-1002.

Nuthmann, A., Vitu, F., Kliegl, R., \& Engbert, R. (in preparation). Failure to replicate the range effect in a simple oculomotor task - implications for eyemovement control in reading.
O'Regan, J. K. (1989). Visual acuity, lexical structure and eye movements in word recognition. In B. Elsendoorn \& H. Bouma (Eds.), Working models of human perception. (pp. 261-292). London: Academic Press.

O'Regan, J. K. (1990). Eye movements and reading. In E. Kowler (Ed.), Eye movements and their role in visual and cognitive processes (pp. 395-453). Elsevier.

O'Regan, J. K. (1992). Optimal viewing position in words and the strategy-tactics theory of eye movements in reading. In K. Rayner (Ed.), Eye movements and visual cognition: Scene perception and reading. New York: Springer-Verlag.

O'Regan, J. K., \& Lévy-Schoen, A. (1987). Eye movement strategy and tactics in word recognition and reading. In M. Coltheart (Ed.), Attention and performance xii: The psychology of reading (pp. 363383). Hillsdale, NJ: Erlbaum.

Ottes, F. P., Van Gisbergen, J. A. M., \& Eggermont, J. J. (1984). Metrics of saccade responses to visual double stimuli - 2 different modes. Vision Research, 25, 849862.

Ottes, F. P., Van Gisbergen, J. A. M., \& Eggermont, J. J. (1985). Latency dependence of colour-based target vs. Nontarget discrimination by the saccadic system. $\mathrm{Vi}$ sion Research, 25(6), 849-862.

Rayner, K. (1979). Eye guidance in reading: Fixation location within words. Perception, 8, 21-30.

Rayner, K., \& Fischer, M. H. (1996). Mindless reading revisited: Eye movements during reading and scanning are different. Perception \& Psychophysics, 58(5), 734-747.

Reichle, E. D., Rayner, K., \& Pollatsek, A. (2003). The e$\mathrm{z}$ reader model of eye movement control in reading: Comparisons to other models. Behavioral and Brain Sciences, 26, 445 - 526.

Reilly, R., \& O'Regan, J. K. (1998). Eye movement control during reading: A simulation of some wordtargeting strategies. Vision Res, 38(2), 303-317.

Reilly, R., \& Radach, R. (2003). Foundations of an interactive model of eye movement control in reading. In J. Hyönä, R. Radach \& H. H. Deubel (Eds.), Cognitive and applied aspects of eye movement research (pp. 429-455). Amsterdam: Elsevier. 
Reilly, R., \& Radach, R. (2006). Some empirical tests of an interactive activation model of eye movement control in reading. Cognitive Systems Research, 7, 34-55.

Rovamo, J., Virsu, V., \& Nasanen, R. (1978). Cortical magnification factor predicts photopic contrast sensitivity of peripheral vision. Nature, 271(5640), 54-56.

Van Rensbergen, J., \& de Troy, A. (1993). A reference guide for the leuven dual-pc controlled purkinje eyetracking system (Technical Report): Laboratory of Experimental Psychology, Catholic University of Leuven.

Vitu, F. (1991a). The existence of a center of gravity effect during reading. Vision Research, 31(7/8), 12891313.

Vitu, F. (1991b). Research note: Against the existence of a range effect during reading. Vision Research, 31(11), 2009-2015.

Vitu, F. (2003). The basic assumptions of e-z reader are not well-founded. Behavioral and Brain Sciences, 26(4), 506-507.

Vitu, F. (2005). Visual extraction processes and regressive saccades in reading. In $\mathrm{G}$. Underwood (Ed.), Cognitive processes in eye guidance. Oxford, NY: Oxford University Press. pp. 1-32.

Vitu, F. \& Casteau, S. (2008). Saccadic eye movements in two-stimulus visual displays: Foveal and peripheral distractor stimuli are not equal. Perception, 35, ECVP Abstract Supplement.

Vitu, F., Lancelin, D., Jean, A., \& Farioli, F. (2006). Influence of foveal distractors on saccadic eye movements: A dead zone for the global effect. Vision Research, 46, 4684-4708.

Vitu, F., Lancelin, D., \& Marrier d'Unienville, V. (2007). A perceptual-economy account for the InvertedOptimal Viewing Position effect. Journal of Experimental Psychology: Human Perception and Performance, 33(5), 1220-1249.

Vitu, F., O'Regan, J. K., Inhoff, A. W., \& Topolski, R. (1995). Mindless reading: Eye-movement characteristics are similar in scanning letter strings and reading texts. Perception and Psychophysics, 57, 352-364.
Viviani, P., \& Swensson, R. G. (1982). Saccadic eye movements to peripherally discriminated visual targets. Journal of Experimental Psychology: Human Perception and Performance, 8(1), 113-126.

Walker, R., Deubel, H., Schneider, W. X., \& Findlay, J. M. (1997). Effect of remote distractors on saccade programming: Evidence for an extended fixation zone. Journal of Neurophysiology, 78(2), 1108-1119.

Weber, H., Latanov, A., \& Fischer, B. (1993). Context dependent modulations of express and regular saccades in man and monkey. Experimental Brain Research, 93, 335-344.

Yang, S.-N. (2006). An oculomotor-based model of eye movements in reading: The competition-interaction model. Cognitive Systems Research, 7, 56-69.

Yang, S.-N., \& McConkie, G. W. (2001). Eye movements during reading: A theory of saccade initiation times. Vision Research, 41, 3567-3585.

Yang, S.-N., \& McConkie, G. W. (2004). Saccade generation during reading: Are words necessary? The European Journal of Cognitive Psychology, 16(1/2).

Zelinsky, G. J., Rao, R. P. N., Hayhoe, M. M., \& Ballard, D. H. (1997). Eye movements reveal the spatiotemporal dynamics of visual search. Psychological Science, 8, 448-453. 